\title{
Personalized Circadian Light: A Digital Siblings Approach
}

\author{
C. Papatsimpa ${ }^{\mathrm{a}, 1}$ and Jean-Paul Linnartz ${ }^{\mathrm{b}}$ \\ ${ }^{a}$ Eindhoven University of Technology, the Netherlands \\ ${ }^{\mathrm{b}}$ Signify, the Netherlands
}

\begin{abstract}
In this paper, we introduce a human-centric lighting control system optimized to support sleep and circadian coordination. We present an approach to optimize lighting that combines a "digital siblings" approach, i.e., a stochastic extension of a digital twin. It estimates and optimizes parameters in experimentally validated models of circadian and sleep regulation with a novel optimization algorithm for optimal timing of light exposure. We acknowledge that people have varying preferences for the lighting levels throughout the day based on their personal preferences and schedules and explicitly include this as a key parameter in our optimization strategy. Our results show that with the suggested lighting schedules, alignment of circadian rhythmicity to the desired sleep-wake schedule can be achieved with minimal disruption to people's daily lives.
\end{abstract}

Keywords. Circadian light, sleep, digital twin, particle filter, personalized model

\section{Introduction}

Human centric lighting that enhances health, performance and well-being has attracted the attention of the lighting industry in the recent years. Although the pathways through which light affects humans have been studied extensively [1], [2] to date little of these insights have been translated into practical lighting control systems. Nonetheless, there is strong evidence that better lighting systems can significantly improve wellbeing and health. Light is the main time cue for the human biological clock. This internal pacemaker regulates a number of physiological systems including hormone production, heart-rate and body temperature, thus influences our daily sleep-wake pattern, alertness and cognitive performance. Evolution has shaped our physiological processes to follow the natural cycle of light and darkness. Yet, indoor life and electric light have muddled our natural light exposure patterns. Nowadays, people are typically exposed to low daytime lighting and receive excessive exposure to light during the night. This un-natural light exposure acutely suppresses melatonin and sleepiness and delays the circadian clock inducing adverse effects for physiology and cognitive performance [3]. Moreover, social demands often oblige us to set an alarm that is out of phase of our propensity rhythm. In fact, a large part of the population is estimated to have social jet-lag [4], that is, circadian rhythms that are out of phase with people's daily schedules. Circadian rhythm sleeping disorders such as insomnia, inefficient sleep and mismatch between

\footnotetext{
${ }^{1}$ Corresponding Author, Charikleia Papatsimpa, Electrical Engineering Department, Signal Processing Systems Group, Eindhoven University of Technology, Groene Loper 3, 5612 AE Eindhoven, The Netherlands; E-mail: c.papatsimpa@tue.nl.
} 
sleep and circadian rhythmicity are associated with adverse mental and physical outcomes [5]. These trends in general population health combined with the indoor lifestyle is one of the triggers for healthy building design to consider human centric lighting. Well-timed artificial light has potential as an effective time cue for the human biological clock [6], [7]. Yet, despite being technologically feasible, wide-scale adaptation of human centric lighting control as a tool that promotes health, well-being and sleep is still lacking. The main barriers that need to be overcome are 1) Precise monitoring of an individual's actual circadian state; the individual differences in how humans perceive lighting, attributed partly to genetic variations in clock genes [8] and environmental influences [9], create a challenge for the translation of circadian research into lighting control. 2) Assessing circadian time using non-clinical and non-invasive sensor data; the current techniques for circadian monitoring are considerably invasive 3) The creation of automated lighting control systems demands optimization algorithms based on quantified models on how humans process the light input that can be executed by automated control systems.

Our recent work in [10] effectively addresses the first two challenges showing the feasibility of circadian phase estimation based on non-invasive light and actigraphy observations. We developed a digital siblings approach based on the statistical framework of a particle filter that not only estimates the circadian state but also adaptively calibrates model parameters to account for inter-individual differences in circadian response to light. We now address the third challenge by proposing an optimization algorithm for optimally adjusting the timing and levels of light exposure. We introduce an iterative optimization algorithm that derives personalized lighting schedules based on quantified models of the circadian and sleep mechanism. Ideally, people are exposed to the natural light-dark cycle, receiving bright daytime light and avoiding light exposure after sunset. Yet, we acknowledge that such lighting schemes are impractical to co-exist with modern lifestyle. After a day at work or school, people typically spend time with family or performing other leisure activities. We thus propose a novel optimization algorithm that takes into consideration personal lighting preferences. We exploit the fact that light is more biologically effective at certain times of the day than others, i.e., depending on when in the circadian trajectory it is administered, light is able to either phase advance or delay the human circadian clock. These phase shifts can be larger or smaller depending on the timing and magnitude of light exposure. The algorithm automatically finds when it is more effective to tune the light levels in order to exert the maximal circadian effect with the least disruption to a person's private life.

\section{Circadian phase estimation}

The problem of setting the light to optimize sleep and circadian functioning prerequisites the accurate estimation of the underlying circadian state and understanding of the mechanisms that regulate sleep in humans. In this section, we briefly review the underlying mathematical models of the circadian pacemaker and sleep regulation and describe the statistical framework we developed to estimate the circadian state using noninvasive light and actigraphy data. 


\subsection{Models of sleep and circadian regulation}

In order to model the sleep mechanism, we adopt the Phillips-Robinson model [11]. According to the model, sleep occurs because of a flip-flop switch between wakepromoting (MA neurons) and sleep-promoting (VLPO neurons) that inhibit each other. Spontaneous wake-up occurs at the time $\left(t_{w_{-} s p}\right)$ that the firing rate of wake-promoting neurons, $Q_{M A}$, surpasses a threshold value $Q_{t h}$, while sleep onset occurs at the time $\left(t_{s_{-} s p}\right)$ that $Q_{M A}$ drops below the threshold value. The neuronal population is described by neuron mean cell body potential dynamics

$$
\begin{gathered}
\tau_{V L P O} V_{V L P O}^{\cdot}+V_{V L P O}=-v_{V L P O-M A} Q_{M A}+D_{V L P O} \\
\tau_{M A} V_{M A}^{\cdot}+V_{M A}=-v_{M A-V L P O} Q_{V L P O}+D_{M A}
\end{gathered}
$$

The mean cell body potential is related to the firing rates of neurons by the firing function

$$
Q_{j}=\frac{Q_{\max }}{1+\exp \left(\frac{\vartheta-V_{j}}{\sigma}\right)}
$$

where $j=V L P O, M A$. Switching between sleep and wake occurs because of a drive to the VLPO that has both homeostatic and circadian components, the homeostatic drive $H$ with sensitivity $v_{v h}$ and circadian drive $C$ with sensitivity $v_{v c}$ respectively,

$$
D_{V L P O}=v_{v c} C+v_{v h} H+D_{0}
$$

The homeostatic component of the drive is the homeostatic sleep pressure $H$ which represents the sleep dept which increases with $Q_{M A}$

$$
\chi \dot{H}+H=\mu_{H} Q_{M A}
$$

while the circadian component $C$ is approximated by

$$
C=0.5(1+0.8 x-0.55 y)
$$

Light input affects sleep by directly steering the circadian component $C$. In this paper, the dynamics of the circadian system are represented using the Jewett-Forger-Kronauer (JFK) model [12]. The circadian oscillation is described as a modified van der Pol oscillator with state variables $x$ and $y$ that oscillate according to

$$
\begin{gathered}
\dot{x}=\frac{\pi}{12}\left[y+\mu\left(\frac{1}{3} x+\frac{4}{3} x^{3}-\frac{256}{105} x^{7}\right)+B\right] \\
\dot{y}=\frac{\pi}{12}\left\{\frac{1}{3} B y-\left[\left(\frac{24}{\tau 0.99729}\right)^{2}+\mathrm{k} B\right] x\right\}
\end{gathered}
$$


Light enters the model as illuminance $I$. After an initial filtering operation, light input is transformed into the light drive signal $B$. The resulting photic drive depends on the ratio of activated photoreceptors $n$ and the state variables $x$ and $y$, suggesting that the circadian system has varying sensitivity to light throughout the day.

$$
\alpha=a_{0} \sqrt{\frac{I}{9500}} \frac{I}{I+100} .
$$

where $\alpha$ describes the rate of photoreceptor activation following light exposure $I$

$$
\begin{gathered}
\dot{n}=60[\alpha(1-n)-\beta n] \\
B=\mathrm{G} \alpha(1-n)(1-\mathrm{b} x)(1-\mathrm{b} y)
\end{gathered}
$$

All model parameters values are listed in Table 1.

Table 1. Model parameter values

\begin{tabular}{cccr}
\hline Circadian process & Value & Homeostatic process & \multicolumn{1}{c}{ Value } \\
\hline$\mu$ & 0.13 & $\mu_{H}$ & $4 \mathrm{nMs}$ \\
\hline$k$ & 0.55 & $v_{v c}$ & $2.9 \mathrm{mV}$ \\
\hline$a_{0}$ & 0.1 & $\chi$ & $4.5 \mathrm{~h}$ \\
\hline$\beta$ & 0.007 & $v_{v h}$ & $1 \mathrm{mVnM}^{-1}$ \\
\hline$G$ & 37 & $D_{0}$ & $10.2 \mathrm{mV}$ \\
\hline$\tau$ & $24.2 \mathrm{~h}$ & $\tau_{v, m}$ & $10 \mathrm{~S}$ \\
\hline$b$ & 0.4 & $v_{M A-V L P O}$ & $2.1 \mathrm{mVs}$ \\
\hline & & $v_{M A-V L P O}$ & $1.8 \mathrm{mVs}$ \\
\hline & & $D_{M A}$ & $1.3 \mathrm{mV}$ \\
\hline
\end{tabular}

The models are based on general population data and model parameter values have been determined by fitting average responses to physiological processes. Skeldon et al. [13] showed that changes in circadian and homeostatic parameters can explain the observed inter-individual differences in the timing and duration of sleep. Since we want to predict individual responses to the light input, we make a plea to consider parameter value characterization in our system design. Given the limited size of our training dataset, and in order to avoid over-fitting, we initially chose the circadian parameter $\tau$ (representing the intrinsic circadian period in humans) to be calibrated based on real user data as there is strong evidence that the impact of light exposure on sleep timing strongly depends on the intrinsic circadian period in humans [14].

\subsection{Digital siblings framework}

To verify and compare the impact of multiple alternative light recipes, developing a digital twin of the human circadian pacemaker mechanism would be attractive. However, a deterministic approach of a digital twin is out of reach for our purpose: as the observations are inherently noisy and incomplete, we need a stochastic approach. We interpret the problem of generating a digital twin as one of characterizing the parameters and estimating the state of the human pacemaker. To this end, the statistical approach of 
a particle filter is an appropriate framework. Instead of a deterministic update of the state of the physical model based on input from the monitoring system, we generate a large set of samples (particles), each representing a possible state, thus consisting of possible values resulting from noisy input and uncertain model parameters. We interpret every particle as a digital sibling of the real human. Siblings slightly differ from each other and from the real human not only in key model parameters, but also in their experiences, such as differences in their exposure to light. These differences represent uncertainties and noise in our ability to monitor the human. That is, we use the particle filter in a way that it accounts for both "nature and nurture" of the siblings, by estimating model parameters and state, respectively. The siblings evolve and re-incarnate: particles are filtered and resampled according to their likelihood given the observed data. During cycles of executing the particle filter algorithm, siblings that have parameters that do not fit the model are discarded and replaced by siblings that inherit parameters (and states) that fit the observations better and (hopefully) converge towards the real human. The particle filter propagation and measurement update operations are schematically presented in Figure 1.



Figure 1. Schematic depiction of particle filter operations. State variables are combined in state vector $\boldsymbol{x}$ and individual-specific parameters are captured in $\vartheta$ with initial probability distribution $p\left(\theta_{0}\right) \cdot p\left(x_{t}^{i} \mid x_{t-1}^{i}, \vartheta_{t-1}^{i}\right)$ represents the prediction update step of the particle filter, realized by evaluating the system dynamics in equations (1)-(11). The term $p\left(\theta_{t}^{i} \mid \theta_{t-1}^{i}, x_{t}^{i}, z_{t}\right)$ represents how the new parameter distribution depends on the (previous) parameter, state, and observation $z_{t}$ at instant $t$.

We begin with an initial particle distribution. 1) Each particle (sibling) is an instantiation of a random variation of initial model parameters drawn from realistic distribution of such parameter values in humans and is exposed to an instantiation of random variation of the measured light exposure drawn from a distribution of the error mechanisms of the light sensor. 2) The prediction step propagates each sibling based on the system's dynamics as described by the set of equations (1)-(11). 3) If a measurement is available, each sibling is evaluated against its fit to observations of the human response (e.g. wakeup time). 4) A resampling operation then discards siblings in the areas of low probability and reincarnates the siblings with parameters and states that have a better fit. This repeating process results in a set of values for the model parameters that update with every new observation. A more elaborate description of the mathematical framework can be found in [10]. This digital twin framework ensures a personalized representation of individual people that is connected and continuously updated by data from the real user. 


\section{Light optimization}

The problem of setting the light to optimize circadian aspects does not fit a traditional control systems approach. The impact of light exposure on the circadian mechanism is not instantaneous but requires evaluation of the process for several hours after the actual timing of light exposure, the history of light exposure of several days also has an influence. Thirdly, user preferences might drive the shape of the optimization, as light that is biologically optimal is not always preferred by the user. Here, we present our solution to this complex optimization problem.

\subsection{User satisfaction}

The circadian impact of light needs to be weighed against immediate acute needs to perform tasks or to feel comfortable. These acute needs depend on the individual light preferences, on the activities performed and possibly on many other aspects. We follow the common observation that each user has its own preferred illuminance level $\xi=$ $\left[\xi_{1}, \ldots, \xi_{N}\right]^{2}$, where we take the preferred illuminance level $\xi_{i}[\mathrm{in} 1 \mathrm{x}]$ to be dependent on the $i$-th hour. Here, $N$ denotes the total duration of the observation. Prior to the tests, users had the possibility to self-select different lighting levels depending on what lighting they found comfortable based on their schedules. We are aware of limitations as human tend not to intervene with light settings unless they are dissatisfied. Nonetheless, lacking better data, we used these observations as being representative for the most preferred illuminance levels throughout the day. The human eye senses brightness approximately logarithmically over a moderate range, thus, following [15], we model user satisfaction for lighting level $I_{i}$ as a log-normal shaped function according to

$$
U\left(I_{i}\right)=\exp \left(-\frac{\left(\ln I_{i}-\ln \xi_{i}\right)^{2}}{2 \sigma^{2}}\right)
$$

Here, parameter sigma $\sigma$ describes the tolerance for illuminance of each individual; some people are only satisfied with a small range of illuminance values while others are more tolerant.

\subsection{Mathematical formulation of the lighting control system}

We consider a dimmable lighting system denoted as $I=\left[I_{1}, \ldots, I_{N}\right]$, where $I_{i}$ is the illuminance level [lx] of the $\mathrm{i}$-th hour, $0 \leq I_{i} \leq I_{\max }$ and $I_{\max }$ corresponds to the illuminance level when the lighting system is fully turned on. The goal is to optimize vector $I$, i.e., the light levels, such that we simultaneously minimize the social jet-lag and lighting discomfort. We quantify social jet-lag as the timing difference between the unconstrained wake-up times $\left(t_{w_{-} s p}\right)$ and the enforced (by social constraints e.g. alarm) wake-up timing $\left(t_{\text {enf }}\right)$, by controlling the light levels in $\boldsymbol{I}$. Given the circadian system dynamics (1)-(11) and the user preference model (12), the lighting control problem may be formulated as a constraint optimization problem as:

\footnotetext{
${ }^{2}$ In this paper, the vectors are represented in bold.
} 


$$
\boldsymbol{w}^{*}=\operatorname{argmin} \quad f(\boldsymbol{I})=\left\|t_{w_{-} s p}-t_{\text {enf }}\right\|
$$

That is subject to:

$$
\begin{gathered}
0 \leq I \leq I_{\max }, \\
U\left(I_{\boldsymbol{i}}\right)>\alpha,
\end{gathered}
$$

Here, $f(\boldsymbol{I})$ is the objective function. The enforced wake-up time $t_{\text {enf }}$ is the time that the user sets his/her alarm, while the spontaneous wake-up time $t_{w_{-} s p}$ is derived by the mathematical model described by the set of Equations (1)-(11), using $I$ as the input in Equation (9). The first constraint is a physical requirement that the dimming level should be between 0 and $I_{\max }$. The second constraint ensures users' comfort by offering satisfying lighting conditions to their preference, i.e., user satisfaction function is not allowed to drop below a certain threshold (e.g., $\alpha=0.6$ ensures that a user is $60 \%$ satisfied with the lighting level).

\subsection{Solution to the optimization problem}

The relationship describing the system dynamics (1)-(11) is highly non-linear, and the impact of light $I$ on the wake-up time $t_{w_{-} s p}$ is not direct, but requires the evaluation of the process for several hours afterwards. As a result, a closed-form expression for $\boldsymbol{I}^{*}$ cannot be found analytically. To solve this, we follow a numerical approach. The iterative optimization algorithm exploits the fact that light is more biologically effective at certain times of the day than others by tuning the light levels at the time slots that are able to introduce larger shifts in the spontaneous wake-up times but exert the least disruption to the user. We use an iterative scheme that operates by introducing a small change to the light level step by step in an appropriately chosen time interval. The underlying principle is to take the interval that additional light has the highest biological impact and least user disruption. Formally, in the $k$-th iteration step, we change the lighting level to the time slot $i_{k}$ that then minimizes the weighted sum of the impact on wake-up time and user disruption, i.e., we chose

$$
i_{k}=\underset{i}{\operatorname{argmin}}(1-\gamma) f\left(I_{i}\right)+\gamma\left(1-U\left(I_{i}\right)\right.
$$

Here, $U\left(I_{i}\right)$ represents the satisfaction of the user for a lighting level $I_{\boldsymbol{i}}$, consequently, minimizing the objective function $1-U\left(I_{i}\right)$ minimizes the disruption of the user. Parameter $\gamma$ weights the input of each objective function. The searching is stopped when the improvement drops below a certain threshold.

\section{Results}

The results presented here are based on data from a field study with 15 participants (average age $70.9 \pm 4$ years). The participants were asked to join the study for 7 days following their normal daily routines. Each participant wore a Philips Actiwatch Spectrum Pro measuring actigraphy (sleep onset and offset times) and light intensity. 
First, we applied the digital sibling framework to the field data in order to estimate the intrinsic circadian period of each participant (model parameter $\tau$ ). Our results confirm the large inter-individual variability in circadian period. In fact, in our limited sample size, the estimated intrinsic circadian period was distributed with $24.12 \mathrm{~h} \pm 0.21$, matching clinically measured distribution of intrinsic circadian period in humans [16]. People do not only show variability in genetic predistortions, e.g., variability in $\tau$, but also in the light levels they are exposed throughout the day. Data from the field study, presented in Figure 2, reveal the large inter- and intra-individual differences in the daily patterns of light exposure, i.e., the timing and magnitude of light exposure varied both between participants, but also from day to day within the same participant. The digital siblings approach results in a personalized to every participant circadian model that enables more accurate predictions of how users respond to light exposure.

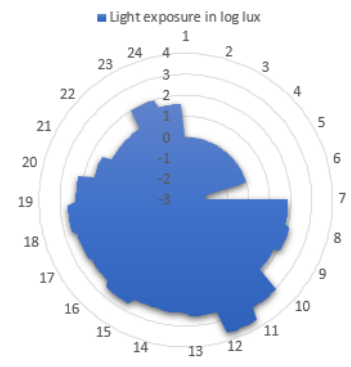

Participant 2, day 1

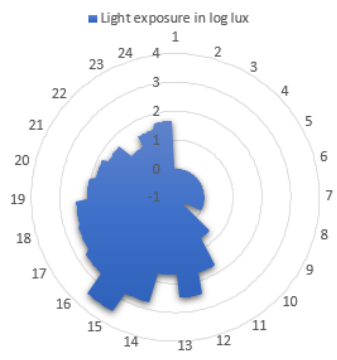

Participant 12 , day 1

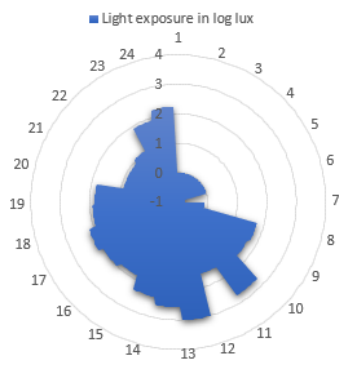

Participant 2, day 2



Participant 12 , day 2

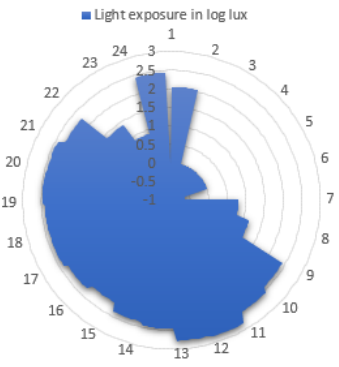

Participant 2, day 7



Participant 12 , day 7

Figure 2. Inter- and intra-subject variability in daily light exposure [in log lux] throughout the 24 hour day. Illustrative examples through polar plots in 2 study participants.

The efficacy of lighting control could potentially be optimized by individual tailoring, based on personal light preferences and considering the internal rhythms of users. As an illustrative example, we present optimization results for a single participant. This participant is characterized by an average intrinsic circadian period ( $\tau=24.21$ ), however, he typically receives bright light during the late evening and night (see Figure 2) which considerably delays his sleep cycle. In fact, the participant typically wakes-up at $8.5 \mathrm{~h} \pm 0.69$ and sleeps at $0.6 \mathrm{~h} \pm 0.23$. If this participant sets an alarm at $07: 30\left(t_{\text {enf }}=\right.$ 7.5 ), this would mean that his biological rhythm is $\sim 1$ hour misaligned with his daily schedule. We thus wish to optimize the lighting levels to correct this circadian misalignment. Since most people spend a large part of their days in indoor public settings, they usually have little ability to control their lighting conditions. We thus 
present optimization results for a domestic setting (early morning and late evening and night) as a light intervention is easier and more practical to realize in such settings. For the simulations, tolerance $\sigma$ is set to 0.6 and the preferred illuminance level $\xi$ is set to the hourly daily average illuminance prior to the lighting intervention. The resulting lighting schedule, presented in Figure 3a, is characterized by increased light levels in the early morning, as soon as the participant wakes up, and dim light levels during the late evening and night, i.e., the time of the day that light introduces the largest delays in the biological clock. As seen in Figure 3b, the new lighting setting is estimated to gradually shift the sleep pattern of the participant, resulting in earlier wake-up and sleep times compared to the sleep schedule without any lighting intervention. In fact, in the last day of the intervention (day 7), the sleep cycle is estimated to be advanced by approximately 48 minutes resulting in the desired wake-up time (alarm at 07:30).

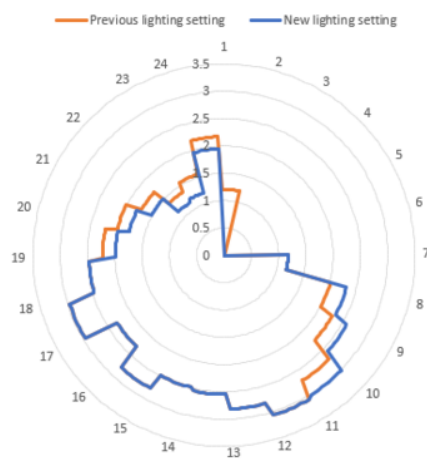

(a)

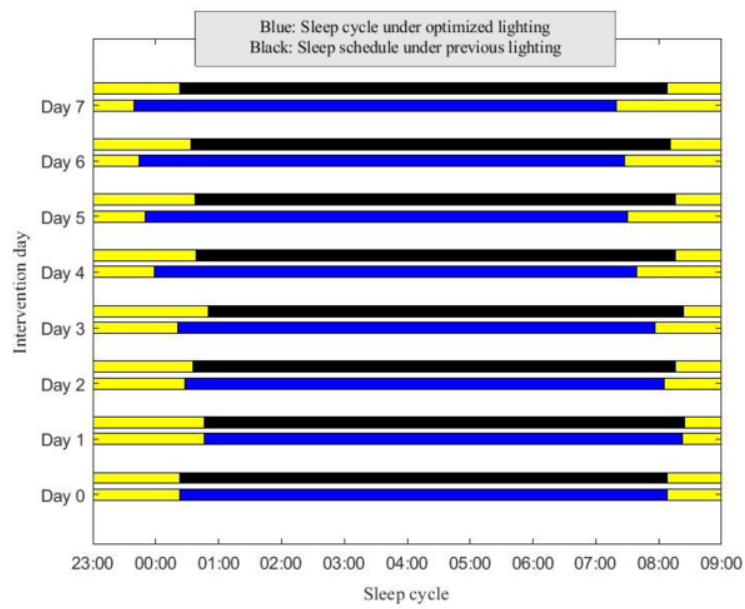

(b)

Figure 3. a) Algorithm suggested lighting schedule superimposed with pro-intervention daily average light exposure. b) Resulting daily shift in sleep cycle compared with sleep pattern prior to the intervention. Results are presented for an example participant with intrinsic circadian period $\tau=24.21$, and tolerance $\sigma=0.6$.

\section{Conclusions}

A well-functioning internal clock is of utmost importance for good sleep and optimal functioning during wake. Disturbances in sleep-wake patterns due to the absence of a strong time cue or an ill-timed signal for the circadian system can result in a lack of energy, cognitive deficits and compromised sleep quality. Despite the insights of how light affects human physiology, the benefits of these insights are not (yet) captured by practical lighting control systems. The natural intra-individual and inter-individual variability of internal time advocates against "one-size-fits-all" lighting interventions and highlights the need for a personalized approach to human-centric lighting. Here, we present a lighting control system optimized to provide personalized lighting that supports sleep and aligns the circadian mechanism to people's daily schedules. Our optimization 
strategy combines a "digital siblings" framework of the circadian mechanism, i.e., a stochastic extension of a digital twin, with a novel optimization algorithm that takes into consideration both the biological effects of light and personal lighting preferences. We arrive at well-timed and feasible lighting schedules that are able to gradually shift the sleep pattern of participants and align their sleep cycles to their daily schedules. Schedules are feasible in the sense that these fit within the personal tolerances to light level deviations.

\section{References}

[1] I. Provencio, I. R. Rodriguez, G. Jiang, W. P. Hayes, E. F. Moreira, and M. D. Rollag, "A novel human opsin in the inner retina," J. Neurosci., vol. 20, no. 2, pp. 600-605, Jan. 2000, doi: 10.1523/jneurosci.20-02-00600.2000.

[2] T. Roenneberg, E. J. Chua, R. Bernardo, and E. Mendoza, "Review Modelling Biological Rhythms," Curr. Biol., vol. 18, pp. 826-835, 9AD, doi: 10.1016/j.cub.2008.07.017.

[3] C. Cajochen et al., "Evening exposure to a light-emitting diodes (LED)-backlit computer screen affects circadian physiology and cognitive performance,” J. Appl. Physiol., vol. 110, no. 5, pp. 14321438, May 2011, doi: 10.1152/japplphysiol.00165.2011.

[4] M. Wittmann, J. Dinich, M. Merrow, and T. Roenneberg, "Social jetlag: Misalignment of biological and social time," in Chronobiology International, 2006, vol. 23, no. 1-2, pp. 497-509, doi: $10.1080 / 07420520500545979$.

[5] K. G. Baron and K. J. Reid, “Circadian misalignment and health,” Int. Rev. Psychiatry, vol. 26, no. 2, pp. 139-154, 2014, doi: 10.3109/09540261.2014.911149.

[6] R. N. Golden et al., "The efficacy of light therapy in the treatment of mood disorders: A review and meta-analysis of the evidence," American Journal of Psychiatry, vol. 162, no. 4. pp. 656-662, Apr2005, doi: 10.1176/appi.ajp.162.4.656.

[7] M. Münch et al., "Blue-Enriched Morning Light as a Countermeasure to Light at the Wrong Time: Effects on Cognition, Sleepiness, Sleep, and Circadian Phase," Neuropsychobiology, vol. 74, no. 4, pp. 207-218, Jul. 2017, doi: 10.1159/000477093.

[8] D. A. Kalmbach et al., "Genetic basis of chronotype in humans: Insights from three landmark gwas," Sleep, vol. 40, no. 2. Associated Professional Sleep Societies,LLC, 01-Feb-2017, doi: 10.1093/sleep/zsw048.

[9] M. Hébert, S. K. Martin, C. Lee, and C. I. Eastman, "The effects of prior light history on the suppression of melatonin by light in humans," J. Pineal Res., vol. 33, no. 4, pp. 198-203, Nov. 2002, doi: 10.1034/j.1600-079X.2002.01885.x.

[10] J. Bonarius, C. Papatsimpa, and J.-P. Linnartz, "Parameter Estimation in a Model of the Human Circadian Pacemaker Using a Particle Filter,” IEEE Trans. Biomed. Eng., pp. 1-1, Sep. 2020, doi: 10.1109/tbme. 2020.3026538 .

[11] A. J. K. Phillips, P. A. Robinson, and A. Phillips, “A Quantitative Model of Sleep-Wake Dynamics Based on the Physiology of the Brainstem Ascending Arousal System," J. Biol. Rhythms, vol. 22, no. 2, pp. 167-179, 2007, doi: 10.1177/0748730406297512.

[12] M. E. Jewett, D. B. Forger, and R. E. Kronauer, "Revised limit cycle oscillator model of human circadian pacemaker.," J. Biol. Rhythms, vol. 14, no. 6, pp. 493-9, Dec. 1999, doi: $10.1177 / 074873049901400608$.

[13] A. C. Skeldon, G. Derks, and D. J. Dijk, "Modelling changes in sleep timing and duration across the lifespan: Changes in circadian rhythmicity or sleep homeostasis?," Sleep Medicine Reviews, vol. 28. W.B. Saunders Ltd, pp. 96-107, 01-Aug-2016, doi: 10.1016/j.smrv.2015.05.011.

[14] A. S. Lazar et al., "Circadian period and the timing of melatonin onset in men and women: Predictors of sleep during the weekend and in the laboratory," J. Sleep Res., vol. 22, no. 2, pp. 155-159, 2013, doi: $10.1111 /$ jsr.12001.

[15] C. Papatsimpa and J.-P. Linnartz, "Personalized Office Lighting for Circadian Health and Improved Sleep,” Sensors, vol. 20, no. 16, p. 4569, Aug. 2020, doi: 10.3390/s20164569.

[16] J. F. Duffy et al., "Sex difference in the near-24-hour intrinsic period of the human circadian timing system,” Proc. Natl. Acad. Sci. U. S. A., vol. 108, no. SUPPL. 3, pp. 15602-15608, Oct. 2011, doi: 10.1073/pnas.1010666108. 